\title{
Physical Comfort of Occupational Exoskeletons: Influence of Static Fit on Subjective Scores
}

\author{
Matteo Sposito \\ matteo.sposito@iit.it \\ Advanced Robotics Department \\ (ADVR), Istituto Italiano di \\ Tecnologia \\ Genoa, Italy \\ Department of Electronics, \\ Informatics and Bioengineerign \\ (DEIB), Politecnico di Milano \\ Milano, Italy \\ Stefano Toxiri \\ Advanced Robotics Department \\ (ADVR), Istituto Italiano di \\ Tecnologia \\ Genoa, Italy \\ stefano.toxiri@iit.it \\ Luigi Monica \\ Department of Technological \\ Innovation and Safety Equipment, \\ INAIL \\ Rome, Italy \\ 1.monica@inail.it
}

\author{
Tommaso Poliero \\ Advanced Robotics Department \\ (ADVR), Istituto Italiano di \\ Tecnologia \\ Genoa, Italy \\ tommaso.poliero@iit.it
}

\author{
Christian Di Natali \\ Advanced Robotics Department \\ (ADVR), Istituto Italiano di \\ Tecnologia \\ Genoa, Italy \\ christian.dinatali@iit.it
}

\author{
Sara Anastasi \\ Department of Technological \\ Innovation and Safety Equipment, \\ INAIL \\ Rome, Italy \\ s.anastasi@inail.it \\ Darwin G. Caldwell \\ Advanced Robotics Department \\ (ADVR), Istituto Italiano di \\ Tecnologia \\ Genoa, Italy \\ darwin.caldwell@iit.it \\ Jesús Ortiz \\ Advanced Robotics Department \\ (ADVR), Istituto Italiano di \\ Tecnologia \\ Genoa, Italy \\ jesus.ortiz@iit.it \\ Francesco Draicchio \\ Department of Occupational and \\ Environmental Medicine, \\ Epidemiology and Hygiene, INAIL \\ Monte Porzio Catone, Rome, Italy \\ f.draicchio@inail.it \\ Elena De Momi \\ Department of Electronics, \\ Informatics and Bioengineerign \\ (DEIB), Politecnico di Milano \\ Milano, Italy \\ elena.demomi@iit.it
}

\begin{abstract}
Exoskeletons come in a variety of shapes and offer different mechanism to adapt to anthropometrics of different users. However, is not always clear how to relate those regulation mechanisms and anthropometric measurement in order to obtain a proper static fit. To this end, in order to maximize kinematic compatibility, we identify a minimal set of anthropometric measurements that affect lower back exoskeleton static fit. Correspondingly, we also provide a definition of static fit specific to lower back exoskeletons. For achieving such results, we present a pilot test of our hypothesis collecting subjective physical comfort evaluation of female and

Permission to make digital or hard copies of part or all of this work for personal or classroom use is granted without fee provided that copies are not made or distributed for profit or commercial advantage and that copies bear this notice and the full citation on the first page. Copyrights for third-party components of this work must be honored

For all other uses, contact the owner/author(s).

Petra '21, June 29 - July 1, 2021, Corfu, Greece

(C) 2021 Copyright held by the owner/author(s)

ACM ISBN 978-1-4503-8792-7/21/06.

https://doi.org/10.1145/3453892.3453995
\end{abstract}

male subjects. The test allows to assess - by means of a custom defined survey -the influence that a customized fit, with respect to a reference fixed fit, has on the score of constructs, regarding perceived pressure, freedom of movement and attachments' stability.

\section{CCS CONCEPTS}

- Human-centered computing $\rightarrow$ Mobile devices; • Computer systems organization $\rightarrow$ Robotics.

\section{KEYWORDS}

Wearable Assistive Device, Subjective Assessment, Occupational Exoskeletons, Physical Interaction

\section{ACM Reference Format:}

Matteo Sposito, Tommaso Poliero, Christian Di Natali, Stefano Toxiri, Sara Anastasi, Francesco Draicchio, Luigi Monica, Darwin G. Caldwell, Elena De Momi, and Jesús Ortiz. 2021. Physical Comfort of Occupational Exoskeletons: Influence of Static Fit on Subjective Scores. In Petra '21: ACM ICPS The PErvasive Technologies Related to Assistive Environments, fune 29 - July 1, 
2021, Corfu, Greeece. ACM, New York, NY, USA, 9 pages. https://doi.org/10. $1145 / 3453892.3453995$

\section{INTRODUCTION}

Occupational exoskeletons are devices intended to provide assistance in tasks that require the workers to sustain unergonomic positions or to move heavy loads or repeatedly handle light ones. Especially in manual material handling $(\mathrm{MMH})$ tasks it is difficult to offer a valid automation solution, that guarantees continuity of service and it is capable to handle goods of several shapes and uneven density. Occupational exoskeletons are a promising solution to address muscular over-extertion that can lead to work-related muskoskeletal disorders (WMSDs). In fact, there are growing positive evidences of exoskeletons' efficacy in decreasing the negative effects on the lumbar [5], [1] and [9] and shoulder muscles [4]. These are the two areas that are stressed the most in industrial tasks [17]. Exoskeletons deliver assistance by mean of passive elements, such as springs or beams [12], and active elements, such as brushless motors.

However, exoskeletons' adoption struggles to rise as it is influenced not only by the efficacy of the device but also by its ergonomics, especially the physical comfort. Indeed, this is influenced by different design choices and aspects of the exoskeletons. In [16], the author underlines the influence of kinematic incompabilities on comfort, while in [14] the authors argue that a versatile exoskeleton, implementing different control strategies in different tasks, enhances users' comfort. Finally, one of the parts that contribute the most to the device ergonomics is the physical attachments: shoulder straps, belts, harnesses, cuffs or any other element that secures an exoskeleton's link to the user's body. Attachments are at direct contact with users' body, transfer assistive forces and maintain the desired position between exoskeleton and human body. Therefore, it is of paramount importance to design and assess physical attachments in an ergonomic way. To this end, we use the concept of static fit, as introduced in [20]. Static fit is not only a matter of sizing based on anthropometrics measurements but it also depends on the postures that the subjects perform during their activities. Moreover, a bad fit implies that the exoskeleton is not aligned with the biological joint of its users and, despite of selfalign mechanisms (SAMs), exoskeleton will generate unpredictable and unwanted reaction forces at the attachments [20] [11]. Those unergonomic forces decrease the efficacy of the exoskeleton and promote migration of the attachments [19].

In this paper we introduce a definition of static fit for a lowerback occupational exoskeleton and perform a subjective evaluation with a custom survey to assess the ergonomics of the attachments, focusing on physical comfort. The constructs of the survey are perceived pressure, freedom of movement and attachment's stability. The introduction of custom tools to evaluate the static fit is expected to improve understanding of human factors requirements on the design phase and help rising adoption of all wearable assistive device. Indeed, the same tools can be applied to attachments for rehabilitation wearable devices, hard or soft exoskeletons and prosthetic devices, like the considerations on static fit in [20]. In Section 2, we briefly introduce, the case study for this paper: the occupational lower-back support exoskeleton XoTrunk. In Section
2.2 we explain the general concept of static fit and expand it with further dimensions for XoTrunk, describing the design approach to guarantee the static fit in relevant postures, namely standing and bending. In 2.3 we report the experimental design and the custom metrics. The protocol is built to resemble most of the activities that are common to MMH tasks. Parameters of the task were chosen to assure a safe activity. For this reason, the NIOSH lifting index is calculated and is below 1 for male and female subjects. In Section 3 we present the results of the subjective survey: single items are presented in descriptive statics ( frequency of occurrence of each rating ), while single constructs and the whole fit score are tested for statistical significance. Finally, in Section 4 and 5 we discuss the static fit results to understand the performance of the device and the limits of this study.

\section{METHODOLOGY}

In this section, we firstly introduce our approach to static fit for a lower-back occupational exoskeleton, XoTrunk, in the context of MMH tasks. Then we present the experimental protocol to test how the static fit affects physical comfort of the users in a laboratory setup, with an active back-support exoskeleton.

\subsection{Occupational exoskeleton XoTrunk}

XoTrunk, in Fig. 1(a), is an occupational active back-support exoskeleton, evolution of Robo-Mate presented in [22]. The system can be worn like a backpack, resting on shoulder and waist and it is also secured to the thighs. The device lowers the stress on the lumbar section of the spine by mean of assistive torques at the level of hip biological joints. Anchor points to unload reaction forces are shoulders, waist and thighs. XoTrunk implements a hierarchical control strategy that, firstly, identifies the activity the user is performing (namely high-level control [14]) then, selects the most appropriate control strategy and generates a desired torque profile based on torso inclination (angle,velocity and acceleration) and forearm myoelectric signals ( mid-level control [21] and [10]) and, eventually, implements torque tracking control algorithms (low-level control [2]).

\subsection{Static fit for a lower-back exoskeleton}

In [20], static fit is defined as the condition where relevant users' and exoskeletons' dimensions match. However, due to large intersubject anthropometrics variability, static fit must be defined for different subject's positions in relevant tasks. Relevant positions for an occupational exoskeleton are standing, walking and bending. XoTrunk's static fit is achieved by mean of: (i) different sizes of physical attachments (i.e. shoulder straps, waist belt and leg wraps), (ii) ergonomic design of the attachments and (iii) fine regulations of their position on the exoskeleton's frame.

Sizes. The different sizes of physical attachments are obtained from anthropometric tables in [13]. The targeted population is males and females in working age (19 - 65 years, from british population); minimum sizes are calulated on th 50 th \%ile of females, maximum sizes are calculated on the 95th \%ile of male population. Data is presented in Table 1. According to an industrial scenario, anthropometrics dimensions are adjusted to account for working clothes ( clad configuration of traditional anthropometry [20]). Therefore, as 


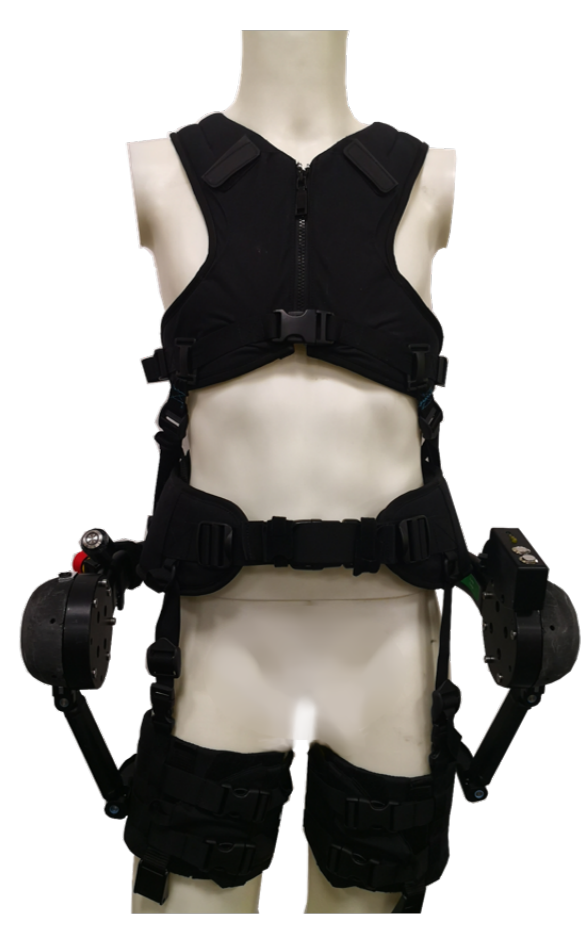

(a)

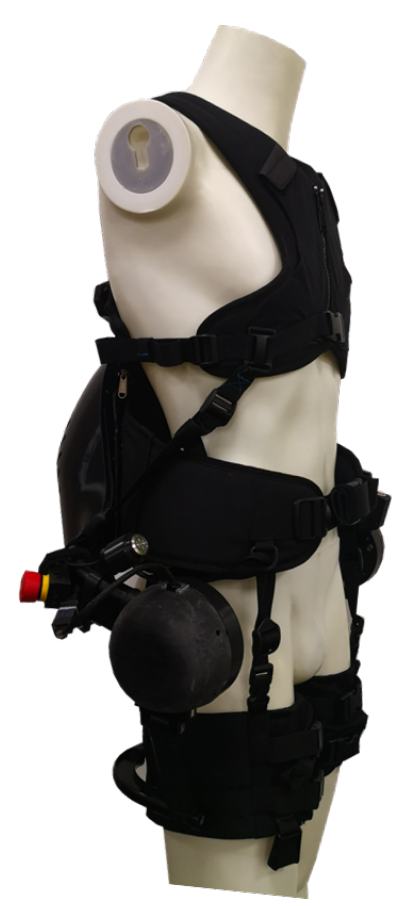

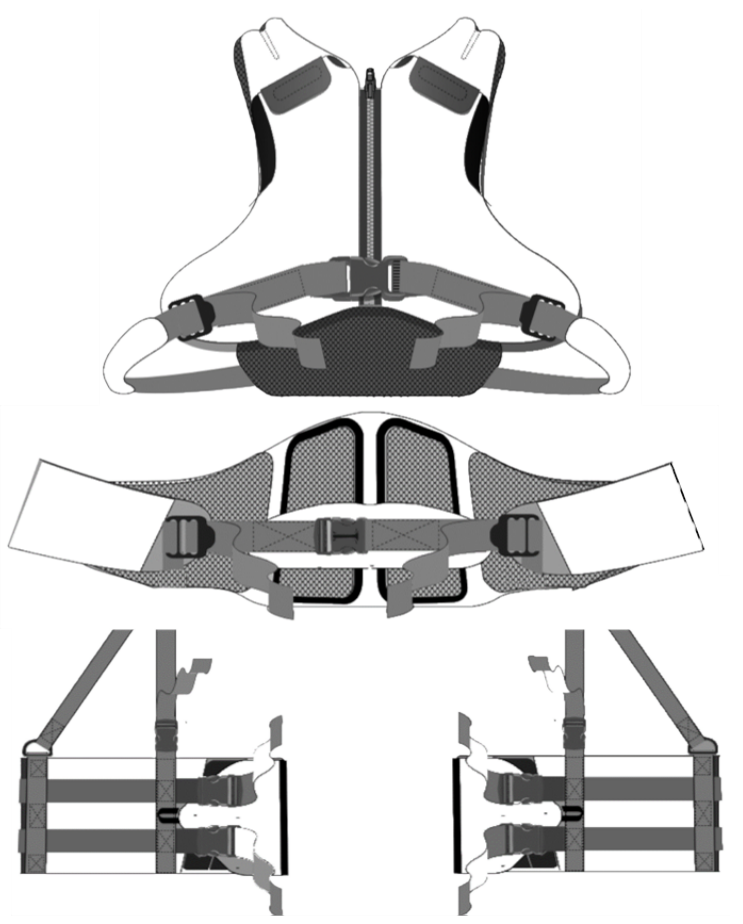

(b)

Figure 1: In (a), a picture of the latest occupational back support exoskeleton, XoTrunk. In (b), drawings of the attachments (from top to bottom): shoulder straps, waist belt and leg wraps.

suggested in [13], 2 to $4 \mathrm{~cm}$ are added to the relevant anthropometric dimensions, that are: chest depth, shoulder breadth (bideltoid), hip width and thigh thickness.

Ergonomic Design. The ergonomic design of physical attachment takes into consideration the particular body shapes where the exoskeleton needs to anchor and needs to unload forces as suggested in [3] and [24]. The ergonomic design for XoTrunk's attachment is implemented by mean of particular shapes, paddings and harness. Shoulder straps, in Fig. 1(b), are designed to secure the weight of the exoskeleton in standing position while the vest-like shape provides an anchor point for the assistive force unloading on the chest in both standing and bending positions. A foam padding is added on the shoulder pads to support the weight on stiff or localized areas like the trapezious and the collarbone. The waist belt, in Fig. 1(b), is designed to secure most of the weight of the exoskeleton on the hips over the iliac crest. To ensure comfort, a $3 \mathrm{~cm}$ padding is used over the bony part. A compliant section it is highly recommended to interface on stiff and bony body parts, lowering the possibility of shear stress on the skin. In addition, the shape of the lumbar cushion is optimized to distribute over a wide area the push on the lumbr muscles in order to obtain a gentle and comfortable pressure. In addition, the compliant material on the belt, meant to ensure comfort, helps the exoskeleton stability ( working as a self-alignment mechanism [11]) but results in rubbing on the lower-back. In the end, the leg wraps belt and shoulder straps are connected by means of an inextensible webbing harness to prevent slippage of the attachments in the two different postures.

Fine adjustements. The fine adjustments are introduced to offer different positions of the attachments on the exoskeleton's frame, to assure the proper fit required by the scope of the device: deliver assistive torque to the hip joints in bending and standing positions during MMH. XoTrunk is composed of a rigid frame with two brushless motors on the sides, at the biological hip joints' level. So, the system requires to unload reaction forces at the shoulder, lower back and thighs. For this reason, to allow the attachments to unload forces effectively, implementing mechanical requirements (e.g. biological and exoskeleton joints alignment, exoskeleton compensation mechanisms [19]), and comfortably, implementing human factors considerations, we identified the best body regions and attachments' shapes as depicted in Fig. 2. Shoulder pads and waist belt are designed to support weight and to unload reaction forces of the exoskeleton, leg wraps are designed only to unload exoskeleton's reaction forces. For the proper static fit, all the attachments are required to be always adherent to the shoulder trapezious region, lower-back lumbar region and thighs in standing and bending positions. To unload assistive forces in bending positions, the shoulder straps should be adherent to the chest to lower the overall pressure caused by assistive forces. To allow that, we added adjustment threaded holes in the attachments reinforcement plates and in the exoskeleton frame, in addition to ladderlocks and webbings, for 
Table 1: Anthropometric data used for sizing of the attachments, from tables in [13]. All dimensions are given in $m m$.

\begin{tabular}{llllllll}
\hline & Height & Chest Depth & Shoulder Breadth & Hip Width & Thigh Thickness & Body Rise & Femur Length \\
\hline Minimum Values & 1610 & 250 & 355 & 370 & 155 & 555 & 385 \\
Maximum Values & 1855 & 285 & 510 & 405 & 185 & 645 & 505 \\
\hline
\end{tabular}

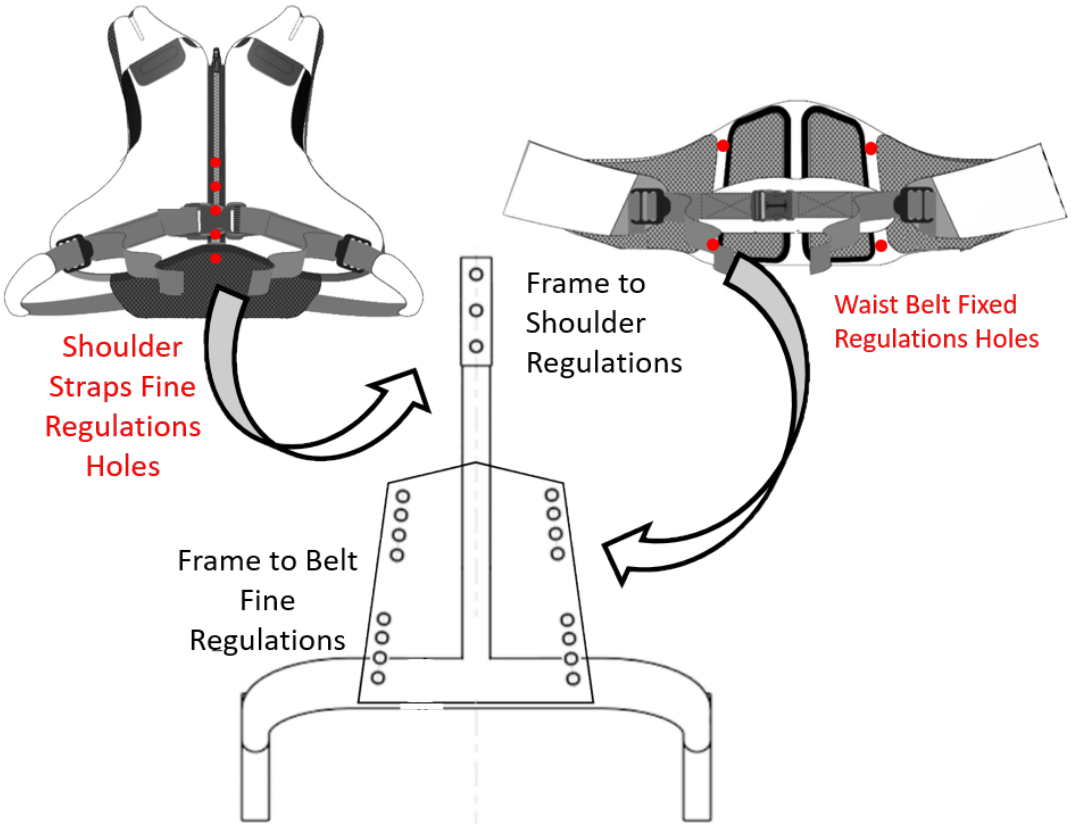

(a)

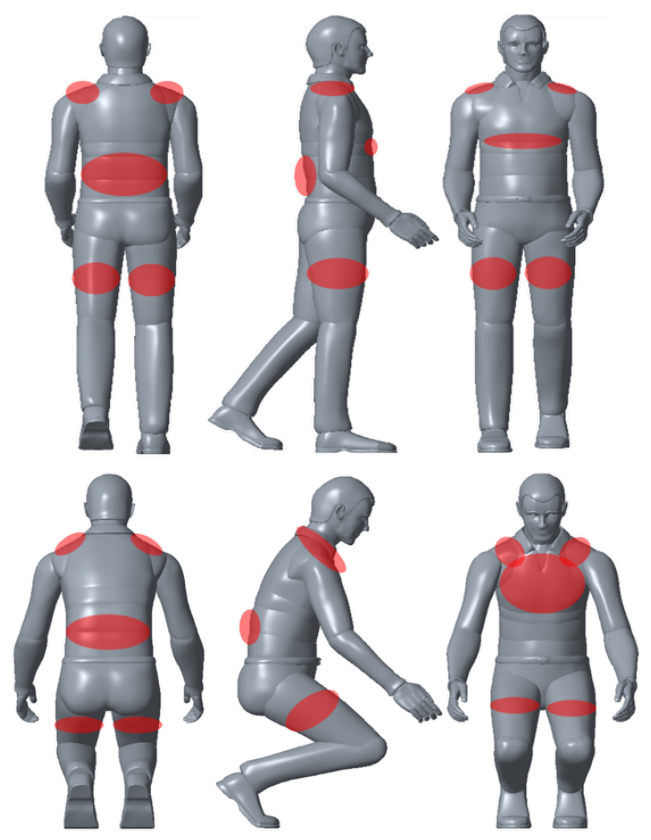

(b)

Figure 2: Fine and coarse adjustements provided for XoTrunk's physical attachments, in (a). The highlighted body areas in (b) show where the attachments must adhere in the positions to assure our definition of proper static fit.

fine adjustments in sizes. In this manner the distance of shoulder pads and waist belt can match the users' body rise.

\subsection{Experimental Design}

The experimental test investigates on the possible correlation of the static fit and the exoskeleton's physical ergonomics. To this end, we define Independent Variables the conditions NOFIT and FIT and Dependent Variables the subjective scores on the following survey constructs: (i) perceived pressure, (ii) freedom of movement, (iii) attachments'stability. The custom survey implements a 7-points fully anchored Likert-like scale. In the NOFIT condition only the sizes of the physical attachments are matched to the subjects, fine regulations are standard for every subject. In the FIT condition, fine regulations are calculated from the subjects' body rise, following the definition of proper static fit in 2.2. The test implements a repeated-measures design without alternating NOFIT|FIT conditions. To avoid any bias from order effects, the subjects have 10 minutes pause ( 5 minute pause plus time to complete the survey) without the exoskeleton in between the two conditions.
Experimental protocol. Ten subjects $(29.6 \pm 4.5$ years, $171.8 \pm 10$ $\mathrm{cm}$ and $67.8 \pm 16 \mathrm{~kg}$ ) divided in five females and five males, volunteered for the tests. Every subject had already experience with other exoskeleton devices. Each subject was instructed on the experimental tasks to perform, all the items of the survey were explained thoroughly. Before starting the test, researchers collected all the anthropometric data of the subjects, the most relevant to sizing are : (i) body rise, (ii) femur length, (iii) thigh circumference, (iv) chest circumference and (v) hip width (all recorded data in Fig. 3(d)). Those measurements are needed to calculate the regulations for the static fit and to choose the right size for the braces.

In order to be as close as possible to a real industrial scenario, the test is composed of lowering, lifting and carrying a load with no restrictions to lifting technique (e.g. squat, stoop or mixed). To resemble an average item that needs to be moved manually (e.g. an item without a regular and solid shape), a sack $L$ was filled with foam and weights for $11.4 \mathrm{~kg}$. This item forces the test subject to carry it without supporting the weight with the abdominal area. The test consists in 10 repetitions of MMH tasks on a test circuit as depicted in Fig. 3(a), an advancement of the protocol presented in 
Table 2: Average anthropometric measurements data from the test subjects. All the measurements are in $\mathrm{mm}$. The data gathered matches with the data used for the physical attachments' design, except for Chest Depth and Shoulder Breadth. Those two measures are aggregated in Chest circumference due to an easier way to collect the measure.

\begin{tabular}{clcllll}
\hline & Height & Chest Circumference & Hip Width & Thigh Width & Body Rise & Femur Length \\
\hline Mean Values & 1718.0 & 921.0 & 388.0 & 168.5 & 480.0 & 477.7 \\
SD & 98.7 & 84.7 & 77.7 & 15.3 & 46.5 & 39.9 \\
\hline
\end{tabular}

[18]. Each cycle lasts 10s, with no restrictions on the duration of each task, and is structured as follows:

- Lifting $L$ from surface S1

- Walk $3.5 \mathrm{~m}$, carrying $L$

- Lower $L$ on surface S2

The NIOSH index [23] of the proposed experiment is 0.81 for male subjects and 0.99 for female subjects. The index is below 1 for all subjects, therefore they are exposed to low risk of injury during the activity. The experiments are compliant with the experimental protocol approved by the Ethical Committee of Liguria (protocol number: 001/2019).

\subsection{Custom Survey}

In [7], subjective comfort of wearable devices are divided in different constructs, called Comfort Descriptors, and the authors present general guidelines to create effective surveys with the Comfort Rating Scales (CRS). Our custom survey, developed following the aforementioned guidelines, focuses on subjective physical comfort constructs, to collect all the feedbacks concerning physical attachments. The Descriptors are: Attachment or physical feel on the body (pressure), Harm or physical effects on the body (friction) and Movement or influence on mobility. The custom survey used in this work is an evolution of the one presented in [18], refined with the feedbacks of pilot subjects during the formative evaluation. Our survey differs from the one introduced by [7], as we propose the following structure:

- Multiple items per construct

- 7-points, fully anchored Likert-like scale for each item.

The aim of this different structure is to focus the attention of the subject on the attachments and to be able to retrieve valuable qualitative information for each single part, as suggested in [3], guiding the subject to provide their score with simple and clear statements and labels for each score.

2.4.1 Data Analysis. As stated in [7] and [8], single fully anchored items should be treated as ordinal non-parametric data (only median is presented), while aggregated data (construct and total fit scores) can be treated as interval non-parametric data (means and standard deviation are presented). In addition, taking into consideration the experimental design, the presented results are tested for statistical relevance with the Wilcoxon Signed Rank test. Whenever the tested item fails the test, we will perform only a descriptive statical analysis.

\section{RESULTS}

We report the analysis with scores presented for each item, construct and the overall score in Table 3. Mean score for the NOFIT condition, $5.0 \pm 1.3$ is $8 \%$ lower than the FIT condition, $5.4 \pm 1.0$. The statistical test succeded to prove a significance difference between the two conditions ( $p$-value $\leq .05)$

Perceived Pressure. Descriptive analysis is reported to show the effects of the two experimental conditions, as the construct fails the statistical relevance test ( $p$-value=.19). In Fig. 4 we report in the first 3 rows the frequencies of the scores for the single items. In the FIT condition, all the items are more positively rated, with respect to NOFIT, the construct rating is $10 \%$ higher in FIT condition (score is $6 \pm 0.8$ ) w.r.t. the NOFIT (score is $5.5 \pm 1.2$ ). The negative scores diminish their impact, in all the items of the construct. The second item shows the biggest difference, among the three. No negative ratings are present for the FIT condition, mostly replaced by the Neutral rating at $23 \%$ with a slight increase of occurencies for all the positive ratings. For the third item we can see a worsening of the negative rating in order, Somewhat Disagree appears in place of Slightly Disagree, but not in occurency, that is always $10 \%$. On the other hand, more subjects gave more positive rating for the thigh pressure in FIT condition, both for magnitude and in occurencies. The first item shows a positive difference for the second condition, even if the only negative rating Slightly Disagree has risen to $20 \%$ in frequency.

Attachments Stability. The same analysis for Perceived Pressure was performed, as the construct fails the statistical relevance test ( $p$-value=.42). In Fig. 4, the reported frequencies of ratings confirm the shared trend that FIT condition get more positive ratings w.r.t. to NOFIT. However, the last item scored the same $20 \%$ of negative ratings but divided in Somewhat Disagree and Slightly Disagree in FIT while in NOFIT the negative score consisted only $20 \%$ of Sligthly Disagree. In addition, the positive score show a mitigation trend with the reduction from $40 \%$, in NOFIT, to $20 \%$ of Completely Agree rating. The item regarding leg wraps' stability shows the best positive trend, moving the average rating towards Somewhat Agree in FIT condition. Even if Completely Agree decreases its frequency from $60 \%$ to $50 \%$ in FIT condition.

Freedom of Movement. This construct fails the statistical relevance test ( $p$-value $=.19)$, the same analysis for the other constructs was performed. In Fig. 4 the last three rows shows the occurrences of the three items of Freedom of Movement against the two conditions. Overall, there is a trend of more positive scores for FIT condition w.r.t. to NOFIT. Indeed, the construct rating is $5.5 \pm 0.9$ for FIT and $5.0 \pm 0.9$ for NOFIT, with a rating increment of $10 \%$ Moreover, the trend is confirmed by the single items' rating with the exception of the second item, that shows a rise of frequency of moderate negative ratings Somewhat Disagree and Slightly Disagree from none to $20 \%$ of the total. In addition, the frequency of mild 


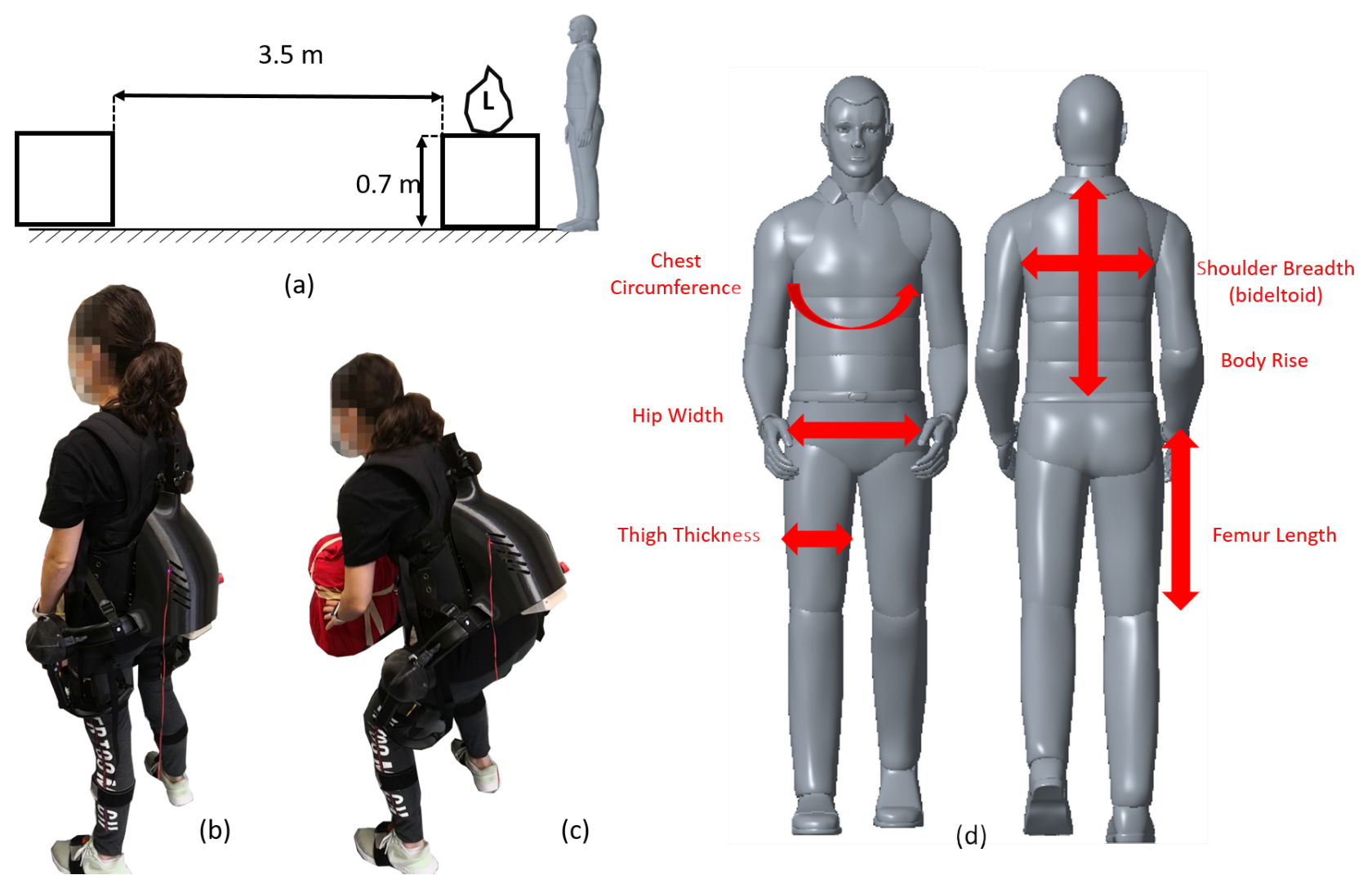

Figure 3: In (a) there is depicted a lateral view of the protocol's setup, with distances and heights of the surfaces where to lift and lower L. In (b) and (c) we show the two different postures, standing and bending, subjects are needed to perform with the exoskeleton to fulfill the task. In (d) we show the anthropometric dimensions that are recorded for every subject.

positive rating Slightly Agree increased from $30 \%$ to $60 \%$, confirming the descending trend.

\section{DISCUSSIONS}

The only statistical relevant score is the one computed from aggregated data from all constructs, Perceived Pressure, Freedom of Movement and Attachments' stability. Despite having relevant clues that the right fine adjustment alignment (as described for XoTrunk in 2.2) influences users' physical comfort, with aggregated data we do not have deep understanding of evolution of each construct to help guiding design choices. Neverthless, we can still retrieve valuable information from descriptive statistics of single items and constructs.

There is a shared trend, among all constructs, that suggest that the physical attachments, their regulations and the exoskeleton's alignment mechanism are effectively fitting on all the users, without distinctions of gender and body sizes and shapes. Indeed, all the constructs improves their average ratings in FIT condition, suggesting that a proper static fit promotes more natural movements and more assistance from the exoskeleton. In fact, from the single items, we can observe that M2 and S2 items (related to assistance delivery) show a trend toward milder positive ratings, suggesting an increment of perceived assistance and its effects.
Constructs Perceived Pressure and Freedom of Movement ratings are the most influenced by the two different conditions of Static Fit, even if statistical relevance tests fail ( $p$-value $=.19)$. Conversely, the ratings for Attachments' Stability are less influenced by the two different conditions ( $p$-value=.42). A possible explanation is that the variation of on-body anchoring position influences directly Freedom of Movement, while influences Perceived Pressure through parasitic reaction forces from kinematic incompatibilities [15] and [11] and different weight unloading. Despite of that, the ratings of Attachments' Stability could suggest that the stability of shoulder pads and belt does not vary in different conditions of alignment. Indeed, the static fit in bending and standing posture of belt and shoulder straps varies because of exoskeletons' structure, as is difficult to obtain always the same fit condition in different postures [20].

Perceived Pressure. There is a shared positive trend, among all items, that suggest pressure distribution is positively influenced by the correct placement on the right body areas [24] achieved through the fine adjustment, the anatomical shapes of the attachments [3] and different sizes. In addition, a bad static fit, leading to worse alignment of exoskeleton and human anthropometrics, can decrease efficacy of the assistance by mean of parasitic reaction forces and cautious unnatural movements performed in tasks (e.g. 
Table 3: Single Items and Constructs statistical results. The values for single items are calculated as medians of the observations for all the subjects in the two conditions. For aggregated scores mean and standard deviations can be computed.

\begin{tabular}{|c|c|c|c|c|c|c|c|}
\hline \multirow[t]{2}{*}{ Constructs } & \multicolumn{2}{|r|}{ Items } & \multicolumn{2}{|c|}{ NOFIT } & \multicolumn{2}{|c|}{ FIT } & \multirow{2}{*}{$\begin{array}{l}\text { Statistics } \\
\text { p-value }\end{array}$} \\
\hline & Labels & Questions & median & SD & median & SD & \\
\hline \multirow{4}{*}{ Perceived Pressure } & P1 & I do not feel pressure on my chest ribs & 5.5 & - & 6.0 & - & - \\
\hline & $\mathbf{P 2}$ & I do not feel pressure on my waist & 5.0 & - & 5.5 & - & - \\
\hline & P3 & I do not feel pressure on my thighs & 5.5 & - & 6.0 & - & - \\
\hline & & Aggregated Scores & 5.1 & 1.2 & 5.7 & 0.8 & .19 \\
\hline \multirow{4}{*}{ Freedom of Movement } & M1 & $\begin{array}{l}\text { I feel free to move and not } \\
\text { encumbered by the attachments } \\
\text { I feel the exoskeleton }\end{array}$ & 5.0 & - & 5.5 & - & - \\
\hline & M2 & $\begin{array}{l}\text { improves my posture } \\
\text { I feel that the assistance during }\end{array}$ & 5.0 & - & 5.0 & - & - \\
\hline & M3 & my task is not bothering me & 6.0 & - & 6.0 & - & - \\
\hline & & Aggregated Scores & 5.0 & 0.9 & 5.3 & 0.9 & .19 \\
\hline \multirow{4}{*}{ Attachments' Stability } & S1 & $\begin{array}{l}\text { I feel shoulder pads are firmly } \\
\text { attached and do not move } \\
\text { I feel the lumbar cushion rubbing }\end{array}$ & 5.0 & - & 5.0 & - & - \\
\hline & S2 & and it feels fine & 5 & - & 5.0 & - & - \\
\hline & S3 & I feel the leg wraps are firmly attached & 6.5 & - & 7 & - & - \\
\hline & & Aggregated Scores & 5.0 & 1.7 & 5.4 & 1.2 & .42 \\
\hline \multicolumn{3}{|c|}{ Static Fit Score } & 5.1 & 1.3 & 5.5 & 1 & .05 \\
\hline
\end{tabular}

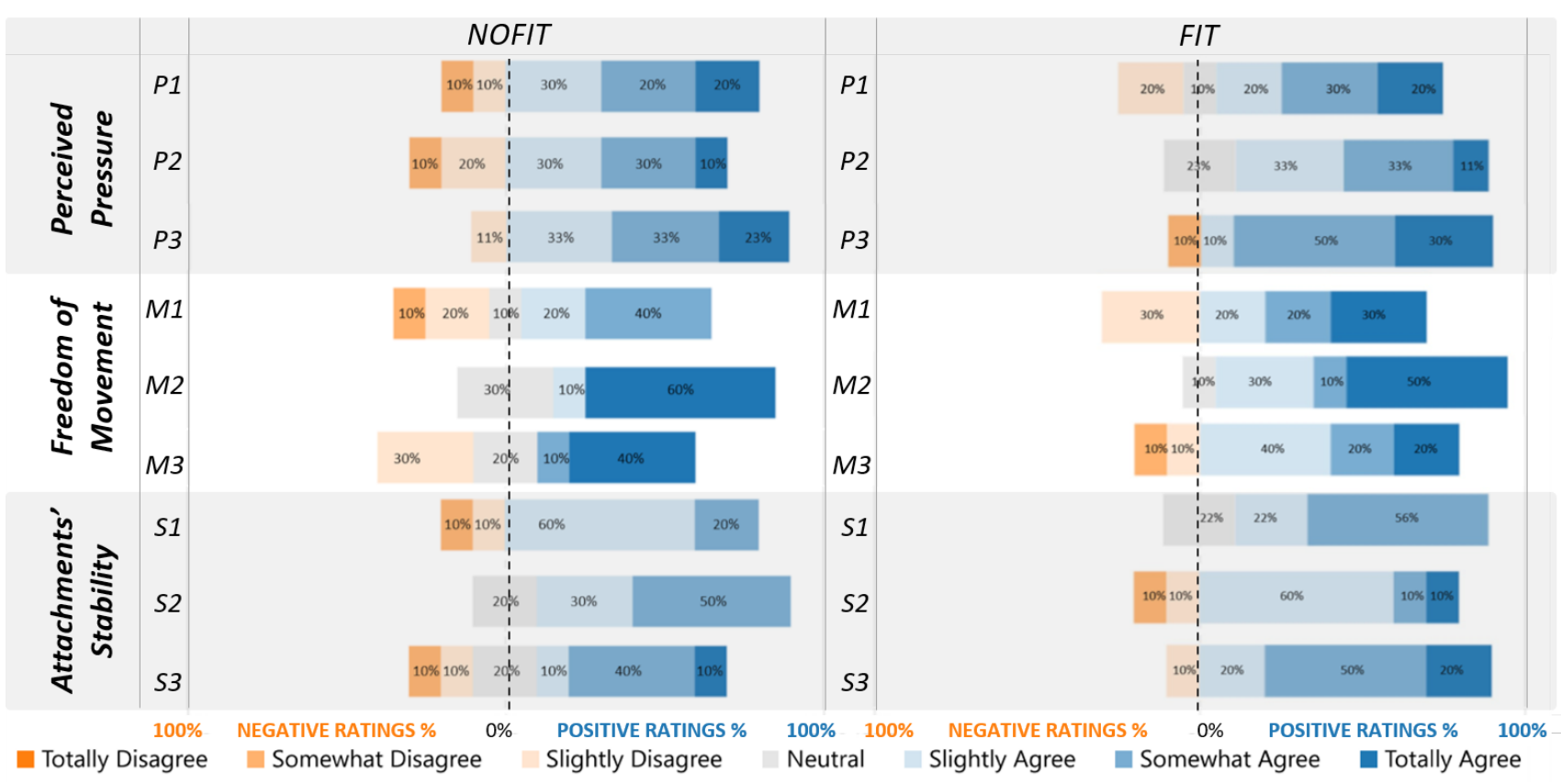

Figure 4: Diverging stacked bar chart of ratings occurences single items for NOFIT (leftmost) and FIT (rightmost) conditions. A diverging stacked bar chart align the bars on a central baseline, and provides 0-100\% ticks for both negative and positve ratings' percentages.

moving the load closer to the hips while carrying decreases the assistance delivered proportional to emg signals). Parasitic forces increase movements of the attachments and unload forces on the attachments in a way that those parts are not designed for, resulting in unbalanced forces loading and focused pressure over small areas (e.g. a rigid cuff can rotate and push only on a small surface), thus leading greater occurrences of negative score in NOFIT condition. We argue that, if the test lasted longer, there would be bigger differences between the two conditions. This is supported by the fact that Perceived Pressure is also proportional to time of exposure and 
frequency of the pressure on the body area ( effect of temporal summation of pain [6]).

Freedom of Movement. Items M1 and M2 show the best positive difference in ratings with respect to $M 3$, thus suggesting that the an ergonomic placement of the attachments promotes more natural movements when wearing the device. Item M3 shows a trend of the ratings to have negative peaks, while centering most of the occurrences in mild positive rating. This effect could be ascribed to more confidence in using the exoskeleton in FIT condition. In fact, if the users move with less caution and in a more natural fashion, they will be assisted with higher torques from the exoskeleton. The assistance provided, depending on the implemented control strategy, could bother the users increasing the frequencies of a mild positive rating.

Attachments Stability. As in Freedom of Movement we find two items, S1 and S3, that show a stronger positive difference in ratings. This suggest that the definition of the proper fit in 2.2 greatly affects the subjective sensations related to the stability of the shoulder straps and leg wraps. Conversely, item S2 shows a different trend, that in general correspond to the same median rating of Slighlty Agree but in condition FIT most of the occurrences move from Somewhat agree to Slighlty Agree. This can be interpreted as an increase of the assistance provided from the exoskeleton. In fact, as explained in 2.2, the lumbar cushion presses and rubs on the lower-back muscles during the assistance phase; the more torque is provided, the more rubbing is experienced. The increase of the assistance from the exoskeleton in FIT can be explained, as before, with an increased confidence in wearing the exoskeleton while it is proper fit.

\subsection{Future Work}

The statistical analysis could benefit of a different protocol approach (such as distance more in time the test in the two conditions for each subject) or, in case of a bigger subject pool size, of a different experimental design. Moreover, a multi-dimensional analysis taking into consideration gender, weight and body shapes could be more informative to identify problems related to shapes and sizes of the attachments. For instance, women usually have more accentuate differences in waist and hip circumferences, thus requiring a different approach to the waist belt and more difficulties in fitting the actual belt. In addition, as suggested in [8], subject's weight influence the perceived sensation of applied pressure and forces on their body. Even if the subjects did not suggest for improvements of wording of the single items, we could introduce more specific instructions: ask to focus on the performance while the subjects are in standing/carrying and bending postures, relevant to static fit definition.

\section{CONCLUSIONS}

This paper presents a custom-defined survey for assessing Perceived Pressure, Attachments' Stability and Freedom of Movement when wearing an occupational exoskeleton, the survey is based on the guidelines presented in [7] and it is an evolution of the one introduced in [19]. We presented an application to assess the static fit (as defined in [20]) of XoTrunk, an occupational exoskeleton for the lower-back that assists in MMH tasks. Results suggest that our implementation of strategies to improve the static fit, that are: sizes, ergonomic design of the attachments and fine adjustments, are effective in scoring more positive ratings in most items of the survey. This preliminary data shows that our approach is positively accepted by subjects of different gender and anthropometric dimensions. However, few subjects feel some negative effects when the exoskeleton delivers the maximum assistance. This suggests that the attachments' design can be improved to deliver assistance. In the end, we argue that a multi-dimensional analysis based on anthropometrics and with more subjects could positively affect the statistical relevance of each construct.

\section{ACKNOWLEDGMENTS}

This work was supported by the Italian Workers' Compensation Authority (INAIL) and Istituto Italiano di Tecnologia (IIT) within the project Sistemi Ciberneteci Collaborativi (SCC) and the STREAM project funded by Shift2Rail Joint Undertaking, established under the European Union's Horizon 2020 framework programme for research and innovation, under grant agreement No 101015418.

\section{REFERENCES}

[1] S. J. Baltrusch, J. H. van Dieën, A. S. Koopman, M. B. Näf, C. Rodriguez-Guerrero, J. Babič, and H. Houdijk. 2019. SPEXOR passive spinal exoskeleton decreases metabolic cost during symmetric repetitive lifting. European fournal of Applied Physiology December (2019). https://doi.org/10.1007/s00421-019-04284-6

[2] Christian Di Natali, Stefano Toxiri, Stefanos Ioakeimidis, Darwin G. Caldwell, and Jesús Ortiz. 2020. Systematic framework for performance evaluation of exoskeleton actuators. Wearable Technologies 1 (2020), 1-25. https://doi.org/10. 1017/wtc. 2020.5

[3] Francine Gemperle, Chris Kasabach, John Stivoric, Malcolm Bauer, and Richard Martin. [n.d.]. Design for Wearability. ([n. d.]).

[4] R. A. R. C. Gopura, K. Kiguchi, and D. S. V. Bandara. 2011. A brief review on upper extremity robotic exoskeleton systems. In 2011 6th International Conference on Industrial and Information Systems. 346-351. https://doi.org/10.1109/ICIINFS. 2011.6038092

[5] Kirsten Huysamen, Michiel de Looze, Tim Bosch, Jesus Ortiz, Stefano Toxiri, and Leonard W. O'Sullivan. 2018. Assessment of an active industrial exoskeleton to aid dynamic lifting and lowering manual handling tasks. Applied Ergonomics 68, November 2017 (2018), 125-131. https://doi.org/10.1016/j.apergo.2017.11.004

[6] Tjasa Kermavnar, Valerie Power, and Leonard O Sullivan. 2017. Computerized Cuff Pressure Algometry as Guidance for Circumferential Tissue Compression. October (2017). https://doi.org/10.1089/soro.2017.0046

[7] James F. Knight and Chris Baber. 2005. A Tool to Assess the Comfort of Wearable Computers. Human Factors: The fournal of the Human Factors and Ergonomics Society 47, 1 (2005), 77-91. https://doi.org/10.1518/0018720053653875

[8] James F. Knight, Daniel Deen-Williams, Theodoros N. Arvanitis, Chris Baber, Sofoklis Sotiriou, Stamatina Anastopoulou, and Michael Gargalakos. 2007. Assessing the wearability of wearable computers. Proceedings - International Symposium on Wearable Computers, ISWC (2007), 75-82. https://doi.org/10.1109/ISWC.2006. 286347

[9] Erik P. Lamers, Juliana C. Soltys, Keaton L. Scherpereel, Aaron J. Yang, and Karl E. Zelik. 2020. Low-profile elastic exosuit reduces back muscle fatigue. Scientific Reports 10, 1 (2020), 1-16. https://doi.org/10.1038/s41598-020-72531-4

[10] Maria Lazzaroni, Stefano Toxiri, Darwin G. Caldwell, Sara Anastasi, Luigi Monica, Elena De Momi, and Jesus Ortiz. 2019. Acceleration-based assistive strategy to control a back-support exoskeleton for load handling: Preliminary evaluation. IEEE International Conference on Rehabilitation Robotics 2019-June (2019), 625-630. https://doi.org/10.1109/ICORR.2019.8779392

[11] Matthias B. Näf, Karen Junius, Marco Rossini, Carlos Rodriguez-Guerrero, Bram Vanderborght, and Dirk Lefeber. 2019. Misalignment Compensation for Full Human-Exoskeleton Kinematic Compatibility: State of the Art and Evaluation. Applied Mechanics Reviews 70, 5 (2019), 050802. https://doi.org/10.1115/1.4042523

[12] Matthias B. Näf, Axel S. Koopman, Saskia Baltrusch, Carlos Rodriguez-Guerrero, Bram Vanderborght, and Dirk Lefeber. 2018. Passive back support exoskeleton improves range of motion using flexible beams. Frontiers Robotics AI 5, JUN (2018). https://doi.org/10.3389/frobt.2018.00072

[13] Stephen Pheasant. 2003. Bodyspace: Anthropometry, Ergonomics, and the Design of Work 
[14] Tommaso Poliero, Lorenza Mancini, Darwin G. Caldwell, and Jesús Ortiz. 2019. Enhancing Back-Support Exoskeleton Versatility based on Human Activity Recognition. 2019 Wearable Robotics Association Conference, WearRAcon 2019 (2019), 86-91. https://doi.org/10.1109/WEARRACON.2019.8719625

[15] Andre Schiele. 2008. An explicit model to predict and interpret constraint force creation in pHRI with exoskeletons. In 2008 IEEE International Conference on Robotics and Automation. IEEE, 1324-1330. https://doi.org/10.1109/ROBOT.2008. 4543387

[16] André Schiele and Frans C T Van Der Helm. 2006. Kinematic design to improve ergonomics in human machine interaction. IEEE Transactions on Neural Systems and Rehabilitation Engineering 14, 4 (2006), 456-469. https://doi.org/10.1109/ TNSRE.2006.881565

[17] Elke Schneider, Xabier Irastorza, European Safety, Sarah Copsey, Health Verjans Lieven Eeckelaert, Véronique Safety, and Belgium. 2010. OSH in Figures: Workrelated Musculoskeletal Disorders in the EU - Facts and Figures.

[18] Matteo Sposito, Darwin G Caldwell, Elena De Momi, and Jesús Ortiz. 2020 (not published yet). Subjective assessment of Occupational Exoskeletons: feasibility study for a Custom Survey for Braces. In International Symposium on Wearable Robotics (WeRob 2020). Springer.

[19] Matteo Sposito, Christian Di Natali, Stefano Toxiri, Darwin G. Caldwell, Elena De Momi, and Jesús Ortiz. 2020. Exoskeleton kinematic design robustness: An assessment method to account for human variability. Wearable Technologies 1
(2020), 1-26. https://doi.org/10.1017/wtc.2020.7

[20] Leia Stirling, Damian Kelty-Stephen, Richard Fineman, Monica L.H. Jones, Byoung Keon Daniel Park, Matthew P. Reed, Joseph Parham, and Hyeg Joo Choi. 2020. Static, Dynamic, and Cognitive Fit of Exosystems for the Human Operator. Human Factors 62, 3 (2020), 424-440. https://doi.org/10.1177/0018720819896898

[21] Stefano Toxiri, Axel S. Koopman, Maria Lazzaroni, Jesús Ortiz, Valerie Power, Michiel P. de Looze, Leonard O'Sullivan, and Darwin G. Caldwell. 2018. Rationale, Implementation and Evaluation of Assistive Strategies for an Active Back-Support Exoskeleton. Frontiers in Robotics and AI 5, MAY (may 2018). https://doi.org/10. $3389 /$ frobt.2018.00053

[22] Stefano Toxiri, Jesus Ortiz, Jawad Masood, Jorge Fernandez, Luis A. Mateos, and Darwin G. Caldwell. 2015. A wearable device for reducing spinal loads during lifting tasks: Biomechanics and design concepts. 2015 IEEE International Conference on Robotics and Biomimetics, IEEE-ROBIO 2015 (2015), 2295-2300. https://doi.org/10.1109/ROBIO.2015.7419116

[23] Thomas R Waters, Vern Putz-Anderson, Arun Garg, and Lawrence J Fine. 1993. Revised NIOSH equation for the design and evaluation of manual lifting tasks. Ergonomics 36, 7 (1993), 749-776.

[24] Clint Zeagler and Clint. 2017. Where to wear it: functional, technical, and social considerations in on-body location for wearable technology 20 years of designing for wearability. Proceedings of the 2017 ACM International Symposium on Wearable Computers - ISWC '17 (2017), 150-157. https://doi.org/10.1145/3123021.3123042 